\title{
EFFICIENCY IMPROVEMENT OF BRIDGE MAINTENANCE AND INSPECTION BY AN UNMANNED AERIAL VEHICLE
}

\author{
J. O. Kim ${ }^{1, *}$ J. K. Lee ${ }^{2}$, K. Kim ${ }^{1}$ \\ ${ }^{1}$ AICT (Advanced Institute of Convergence Technology), Suwon, South Korea - (geostar1, fehouse)@ snu.ac.kr \\ ${ }^{2}$ KICT (Korea Institute of Civil Engineering and Building Technology), Goyang, South Korea - jaekang.lee@kict.re.kr
}

Commission VI, WG VI/4

KEY WORDS: Bridge Maintenance and Inspection, UAV Photogrammetry, Crack Detection, Water Leak, White Coat

\begin{abstract}
:
The economic development and infrastructure of a nation are closely interrelated. In addition, public trust in national infrastructure facilities is closely linked to the preservation of the advantages provided by these facilities to the public. Current maintenance works face certain limitations caused by various reasons: insufficient budget, increasing number of infrastructure facilities requiring maintenance, shortage of manpower, and rapidly increasing number of aging infrastructure facilities. To overcome these limitations, a new approach is required that is different from manual inspection methods under the existing rules and regulations. In this context, this study aimed to explore the efficiency of bridge inspection and maintenance by Unmanned Aerial Vehicles (UAVs) that could observe inaccessible areas, could be conveniently and easily controlled, and could offer high economic benefits. For which, various tests were performed on elevated bridges, and suitable UAV images were obtained. The obtained UAV images were inspected by using machine vision technology, thereby excluding subjective evaluations by humans. Methods for enhancing the objectivity of the inspection were also discussed. The test results showed that both the efficiency and objectivity of the proposed UAV-based method were better efficient than those of the existing bridge maintenance and inspection methods.
\end{abstract}

\section{INTRODUCTION}

The infrastructures in South Korea that were largely built during rapid economic growth in the 1970s and 1980s are more than 30 years old and their deterioration rate exceeds $11.1 \%$. If the old infrastructures are not maintained and inspected systemically and periodically, the efforts to build a national safety net will be challenged greatly. Reducing people's safety concern about the old infrastructures and responding to an evolving technological environment systemically requires efforts of the related field and continued investment in the long run. Entering the era of deteriorating infrastructures, the country is expected to have 9,576 bridges $(30.9 \%)$ that are older than 30 years in a decade and 21,737 bridges $(70.2 \%)$ in two decades as of December 2015. In the US, 503 bridges collapsed in the last 11 years and 250 trillion won was spent for their management. The Ministry of Land, Infrastructure, Transport and Tourism of Japan established a long-term plan to conduct regular inspection on urban infrastructures and bridge maintenance. Their performance and life should be improved and financial burden should be eased through systemic maintenance and inspection from the long-term perspective and the public safety and service quality should be enhanced to effectively address the rapid increase of aging bridges.

\section{BRIDGE MAINTENANCE AND INSPECTION BY AN UNMANNED AERIAL VEHICLE}

This study investigated ways to carry out efficient bridge maintenance and inspection with UAVs that have the potential to address such limits and challenges, enable regular monitoring, bring economic benefit and facilitate operation. To achieve these goals, Test Bridge was considered, obtained UAV images and performed a $3 \mathrm{D}$ modeling based on the UAV image- analysis. Furthermore, a 3D viewer was created to help bridge inspectors intuitively understand and inspect bridges. An inspection checklist is to enable the bridge maintenance and inspection through the $3 \mathrm{D}$ viewer based on the UAV images by referring to the existing bridge inspection checklists. The bridge maintenance and inspection method using the UAV images is analyzed and compared with the existing methods and the economic feasibility of the proposed method is analyzed.

\section{CONCLUSION}

This study found that the quality of the UAV images significantly affected the entire bridge inspection and regular updates at certain times should be possible due to the nature of the inspection work. This implied that producing homogeneous image quality with the existing images could be the key to an efficient UAV-based inspection. To achieve this goal, a UAV operation manual and work regulation must be created in the future.

\section{ACKNOWLEDGEMENTS}

This work was supported by the National Research Foundation of Korea(NRF) grant funded by the Korea government(MSIT) (No. 2019R1A2C1010976).

\section{REFERENCES}

Lee, J.K., Kim, J.O., Park, S.J, 2019: A study on the UAV image-based efficiency improvement of bridge maintenance and inspection. Journal of Intelligent and Fuzzy System 36(2019), 967-983.

Revised July 2019 